\title{
Density correlations in the half-filled Hubbard model
}

\author{
Fabian H. L. Eßler \\ Department of Physics, Theoretical Physics, Oxford University, 1 Keble Road, Oxford OX1 3NP, United Kingdom \\ Holger Frahm \\ Institut für Theoretische Physik, Universität Hannover, D-30167 Hannover, Germany
}

(Received 4 March 1999)

\begin{abstract}
We consider density-density correlations in the one-dimensional Hubbard model at half filling. On intuitive grounds one might expect them to exhibit an exponential decay. However, as has been noted recently, this is not obvious from the Bethe Ansatz/conformal field theory (BA/CFT) approach. We show that by supplementing the BA/CFT analysis with simple symmetry arguments one can easily prove that correlations of the lattice density operators decay exponentially. [S0163-1829(99)12135-0]
\end{abstract}

Recently, density correlation functions have been studied for various multicomponent one-dimensional electron systems within the framework of the bosonization approach., An important issue raised in these works is the question whether or not these correlators exhibit a power-law behavior at large distances in the presence of a Mott-Hubbard gap (see also Refs. 3 and 4). For the particular case of the half filled, one-dimensional Hubbard model a bosonization analysis yields the expected exponential decay, which at first sight appears to be at odds with the results obtained from the Bethe Ansatz ${ }^{5}$ (see, e.g., Ref. 20 of Ref. 1).

In one spatial dimension, the asymptotic behavior of correlation functions can be analyzed by means of the BetheAnsatz/conformal field theory (BA/CFT) approach. Furthermore, all possible critical dimensions can be obtained from the exact solution. ${ }^{5}$ Since the expansion of the lattice operators in terms of the conformal fields is not known explicitly, the resulting expressions contain unknown amplitudes. In particular, the leading term in the BA/CFT expression for the correlation function under question could actually vanish. This is the origin of the controversy mentioned above. In certain cases, including the one discussed here, it is possible to resolve this issue by employing symmetry considerations of the underlying microscopic model.

In this note, we are interested in connected correlation functions of electron densities at half filling. Using the selection rules of conservation of numbers of electrons with spin up and down, one can show from the exact solution (specialized to the case of zero-magnetic field) that

$$
\begin{aligned}
G_{n n}(t, x) & =\langle[n(t, x)-\langle n\rangle][n(0,0)-\langle n\rangle]\rangle \\
& \rightarrow A_{1} \frac{\cos \left(\pi x / a_{0}\right)}{|x+i \mathrm{v} t|}+A_{2} \frac{x^{2}-\mathrm{v}^{2} t^{2}}{\left(x^{2}+\mathrm{v}^{2} t^{2}\right)^{2}}+\cdots
\end{aligned}
$$

where $\mathrm{V}$ is the spin velocity, $a_{0}$ is the lattice spacing and $A_{1,2}$ are unspecified amplitudes that depend on the physical parameters of the problem, i.e., interaction strength and in the general case magnetic field. Note that in Eq. (1) we have omitted similar, subdominant terms.

We will now show that by using the full set of selection rules for the half-filled Hubbard model, one can establish that the amplitudes of all contributions to Eq. (1), that decay algebraically, are identically zero and that the correlator thus decays exponentially.

The Hamiltonian of the half-filled Hubbard model is of the form

$$
\mathcal{H}(U)=-\sum_{\langle i j\rangle}\left\{c_{i \sigma}^{\dagger} c_{j \sigma}+\text { H.c. }\right\}+U \sum_{i}\left(n_{i \uparrow}-\frac{1}{2}\right)\left(n_{i \downarrow}-\frac{1}{2}\right) \text {. }
$$

Here $c_{i \sigma}, c_{i \sigma}^{\dagger}$ are canonical annihilation and creation operators for electrons with spin $\sigma$ on site $i, n_{i}=\Sigma_{\sigma} n_{i \sigma}$ $=\Sigma_{\sigma} c_{i \sigma}^{\dagger} c_{i \sigma}$ is the number operator density for electrons.

In addition to the $S U(2)$ invariance in the spin degrees of freedom the Hubbard Hamiltonian on a bipartite lattice commutes with the generators of a second $S U(2)$ related to particle-hole symmetry. ${ }^{6,7}$ In the one-dimensional case, the local generators of this $\eta$-pairing $S U(2)$ are

$$
\begin{gathered}
\eta_{j}^{+}=(-1)^{j} c_{j \uparrow} c_{j \downarrow}, \\
\eta_{j}^{-}=\left(\eta_{j}^{+}\right)^{\dagger}, \quad \eta_{j}^{z}=\frac{1}{2}\left(1-n_{j}\right),
\end{gathered}
$$

satisfying commutation relations $\left[\eta_{j}^{ \pm}, \eta_{j}^{z}\right]= \pm \eta_{j}^{ \pm}$and $\left[\eta_{j}^{+}, \eta_{j}^{-}\right]=2 \eta_{j}^{z}$. Combining the two symmetries one obtains the well-known $S U(2) \otimes S U(2) / Z_{2}=S O(4)$-symmetry of the Hubbard model.

At half filling, the ground state $|\Omega\rangle$ is an $S O(4)$ singlet. ${ }^{8,9}$ Denoting by $n(t, x)$ the density operator at $x=a_{0} j$ and time $t$ we want to determine the asymptotic behavior for $j \rightarrow \infty$ of the connected correlation function

$$
\begin{aligned}
G_{n n}(t, x) & =4\left\langle\eta^{z}(t, x) \eta^{z}(0,0)\right\rangle \\
& =4 \sum_{n}\left\langle\Omega\left|\eta^{z}(t, x)\right| n\right\rangle\left\langle n\left|\eta^{z}(0,0)\right| \Omega\right\rangle \\
& =4 \sum_{n} \exp \left[i\left(\epsilon_{n} t-k_{n} x\right)\right]\left|\left\langle n\left|\eta^{z}(0,0]\right| \Omega\right\rangle\right|^{2} .
\end{aligned}
$$

Hence, the matrix elements 


$$
\left\langle n\left|\eta_{1}^{z}\right| \Omega\right\rangle
$$

determine which intermediate states will contribute to the asymptotic behavior of Eq. (4).

In the BA/CFT approach to correlation functions in integrable models one usually proceeds as follows: Firstly, one calculates the finite-size scaling behavior of the energies $\epsilon_{n}$ and momenta $k_{n}$ of the low-lying states entering the expression (4). Secondly, one uses the quantum numbers of the operator entering the matrix element to eliminate "incompatible" intermediate states. Finally, the CFT relation between finite-size energies and scaling dimensions is used to obtain the asymptotic (power law) behavior of correlation functions by summing over the remaining low-lying intermediate states.

In Ref. 5 only particle numbers for spin- $\sigma$ electrons were used in the second step. This corresponds to taking into account only quantum numbers associated with the Cartan generators $S^{z}$ and $\eta^{z}$ but not those with the total spins $\vec{S}^{2}$ and $\vec{\eta}^{2}$. As a result, one obtains Eq. (1), which seems to indicate that $G_{n n}$ decays algebraically for large distances in spite of the presence of a charge gap at half filling. We will now show, by considering the full set of $S O(4)$ quantum numbers, that only intermediate states with a gap contribute to Eq. (5) and the density correlations decay exponentially.

Let us calculate the total $\eta$-spin quantum number of the state $\eta_{1}^{z}|\Omega\rangle$ : using the commutation relations between the $\eta$-pairing operators [and the fact that $|\Omega\rangle$ is a singlet under the $\eta$-pairing $\mathrm{SU}(2)]$ one easily obtains

$$
\vec{\eta}^{2}\left(\eta_{1}^{z}|\Omega\rangle\right)=\left[\vec{\eta}^{2}, \eta_{1}^{z}\right]|\Omega\rangle=2\left(\eta_{1}^{z}|\Omega\rangle\right),
$$

showing that this state is a triplet of the $\eta$-pairing $S U(2)$. As a consequence, only intermediate states that are $\eta$-pairing triplets can contribute to the correlation function (4).

As is well known only pure spin excitations are gapless in the repulsive half-filled Hubbard model. However, these are all singlets of the $\eta$-pairing $S U(2) .{ }^{9}$ Therefore the corresponding matrix elements (5) vanish identically and cannot contribute in $G_{n n}$. We conclude that the lowest energy intermediate states with nonzero matrix elements (5) are holon-antiholon scattering states with energy above the Mott-Hubbard gap. As a result the density-density correlation function exhibits exponential decay at large distances for any positive $U$

$$
G_{n n}(t, x) \rightarrow \exp (-\alpha x), \quad x \rightarrow \infty .
$$

The determination of $\alpha(U)$ is an interesting open problem. On general grounds we expect it to be proportional to the holon gap, which is given by ${ }^{10,11}$ (see also Ref. 12)

$$
\begin{aligned}
\Delta(U) & =\frac{U}{2}-2+4 \sum_{n=1}^{\infty}(-1)^{n}\left[\sqrt{1+(n U / 2)^{2}}-n U / 2\right] \\
& \sim \frac{4}{\pi} \sqrt{U} \exp (-2 \pi / U), \quad \text { for } U \rightarrow 0 .
\end{aligned}
$$

If one considers correlation functions of "point-split" densities such as $N_{j}^{(\mathrm{ps})}=\Sigma_{\sigma} c_{j \sigma}^{\dagger} c_{j+1, \sigma}$ the above symmetry argument does not imply the vanishing of, e.g., the matrix element $\left\langle\mathrm{ST}\left|N^{(\mathrm{ps})}(0,0)\right| 0\right\rangle$, where $|\mathrm{ST}\rangle$ denotes a spin-triplet excitation. ${ }^{13}$ Given that symmetry does not force such matrix elements to vanish we expect them to be nonzero, which then immediately implies an algebraic decay like in Eq. (1).

We emphasize, that to establish the exponential decay of $G_{n n}$ in the above way it is essential that: (1) There is an exact symmetry in the charge sector of the microscopic Hamiltonian (2). In general this symmetry may not be obvious (as is the case for the Hubbard model). (2) The ground state is a singlet of the corresponding algebra. Note that the above considerations still hold in the presence of a magnetic field as the ground state (in the half-filled band) remains a singlet of the $\eta$-pairing SU(2). (3) All charged (nonsinglet) excitations are gapped.

We note that these conditions are fulfilled for the halffilled Hubbard model on a bipartite lattice in any dimension, provided that $U$ is larger than the critical Mott-Hubbard value. $^{8}$

Analogous conclusions can be reached for spin-spin correlations in the attractive Hubbard model by employing discrete symmetries of the Hamiltonian (2). Under the particlehole transformation for spin up

$$
c_{j, \uparrow} \rightarrow(-1)^{j} c_{j, \uparrow}^{\dagger}, \quad c_{j, \downarrow} \rightarrow c_{j, \downarrow}
$$

the Hamiltonian (2) transforms according to $\mathcal{H}(U)$ $\rightarrow \mathcal{H}(-U)$, whereas $\eta$-pairing and spin $\mathrm{SU}(2)$ symmetries are interchanged $S^{a} \leftrightarrow \eta^{a}, a=+,-, z$. Furthermore, the ground state of the attractive Hubbard model is a spin singlet. This implies that spin-spin correlation functions in the attractive Hubbard model decay exponentially at large distances

$$
\left\langle S^{z}(t, x) S^{z}(0,0)\right\rangle \rightarrow \exp (-\beta x), \quad x \rightarrow \infty,
$$

where $\beta>0$ for any $U<0$.

We are grateful to the Max-Planck-Institut für Physik komplexer Systeme at Dresden for hospitality and to A. O. Gogolin and A. Schadschneider for important discussions. F.H.L.E. was supported by the EPSRC. H.F. was supported in part by the Deutsche Forschungsgemeinschaft under Grant No. Fr $737 / 2-3$.
${ }^{1}$ V. J. Emery, S. A. Kivelson, and O. Zachar, Phys. Rev. B 59, 15 641 (1999).

${ }^{2}$ P. Azaria, A. O. Gogolin, P. Lécheminant, and A. A. Nersesyan, Phys. Rev. Lett. 83, 624 (1999); R. Assaraf, P. Azaria, M. Caffarel, and P. Lécheminant, Phys. Rev. B. 60, 2299 (1999).
${ }^{3}$ V. J. Emery, in Highly Conducting One-Dimensional Solids, edited by J. T. Devreese, R. P. Evrard, and V. E. van Doren (Plenum, New York, 1979).

${ }^{4}$ J. Voit, J. Phys. C 21, L1141 (1988).

${ }^{5}$ H. Frahm and V. E. Korepin, Phys. Rev. B 42, 10553 (1990). 
${ }^{6}$ O. J. Heilmann and E. H. Lieb, Ann. (N.Y.) Acad. Sci. 172, 583 (1971).

${ }^{7}$ C. N. Yang, Phys. Rev. Lett. 63, 2144 (1989).

${ }^{8}$ E. H. Lieb, Phys. Rev. Lett. 62, 1201 (1989).

${ }^{9}$ F. H. L. Eßler and V. E. Korepin, Phys. Rev. Lett. 72, 908 (1994); Nucl. Phys. B 426, 505 (1994).
${ }^{10}$ E. H. Lieb and F. Y. Wu, Phys. Rev. Lett. 20, 1445 (1968).

${ }^{11}$ A. A. Ovchinnikov, Zh. Éksp. Teor. Fiz. 57, 2137 (1969) [Sov. Phys. JETP 30, 1160 (1970)].

${ }^{12}$ C. A. Stafford and A. F. Millis, Phys. Rev. B 48, 1409 (1993).

${ }^{13}$ F. Woynarovich, J. Phys. C 16, 5293 (1983). 